TITLE:

\title{
Nanoscale ablation on patterned diamondlike carbon film with femtosecond laser pulses
}

$\operatorname{AUTHOR}(S)$ :

Miyaji, G; Miyazaki, K

\section{CITATION:}

Miyaji, G ...[et al]. Nanoscale ablation on patterned diamondlike carbon film with femtosecond laser pulses. APPLIED PHYSICS LETTERS 2007, 91(12): 123102.

ISSUE DATE:

2007-09-17

URL:

http://hdl.handle.net/2433/50399

\section{RIGHT:}

Copyright 2007 American Institute of Physics. This article may be downloaded for personal use only. Any other use requires prior permission of the author and the American Institute of Physics. 


\title{
Nanoscale ablation on patterned diamondlike carbon film with femtosecond laser pulses
}

\author{
Godai Miyaji $^{\text {a) }}$ and Kenzo Miyazaki \\ Advanced Laser Science Research Section, Institute of Advanced Energy, Kyoto University, Gokasho, Uji, \\ Kyoto 611-0011, Japan
}

(Received 3 July 2007; accepted 24 August 2007; published online 17 September 2007)

\begin{abstract}
The authors have studied the origin of nanostructure formation on diamondlike carbon film in femtosecond laser ablation at low fluence. Using the thin film target patterned with submicrometer-size stripes, they have observed that the nanostructure starts to be formed on the crest of stripes along the direction perpendicular to the laser polarization. The experimental results have shown that nanoscale ablation for the nanostructuring would preferentially be initiated by the enhancement of localized electric field on the stripe surface with high curvature. (C) 2007 American Institute of Physics. [DOI: 10.1063/1.2784966]
\end{abstract}

Recent experiments of femtosecond laser ablation for solid targets have demonstrated that the periodic structures created on the material surface are often much smaller than the laser wavelength $\lambda^{1-13}$ being in contrast to the so-called ripples with the period close to $\lambda .{ }^{14}$ Such nanostructures have usually been produced at fluence around the ablation threshold. For example, the structure size as small as $\sim \lambda / 10$ or $30 \mathrm{~nm}$ was observed on a diamondlike carbon (DLC) film with superimposed femtosecond pulses at fluence less than the single-pulse ablation threshold. ${ }^{3}$ Similar fine periodic structures, typically less than $\sim \lambda / 3$, have been reported with various kinds of materials such as $\mathrm{BaF}_{2}, \mathrm{CaF}_{2},{ }^{2}$ diamond, ${ }^{4}$ TiN, ${ }^{1,3} \mathrm{AlN},{ }^{7} \mathrm{CrN},{ }^{9} \mathrm{GaN},{ }^{11} \mathrm{SiC},{ }^{6,713} \mathrm{Si}^{8}{ }^{8} \mathrm{GaP}, \mathrm{GaAs},{ }^{5} \mathrm{InP},{ }^{5,10}$ and $\mathrm{ZnSe},{ }^{12}$ where the ablation traces are usually oriented to the direction perpendicular to the laser polarization.

The nanostructuring on solid surface is attractive for emerging applications in nanoscience, since it provides a promising approach to the laser precise material processing beyond the diffraction limit of light. For the development of laser nanoprocessing, one of the most important subjects to be studied is the exact physical process of nanostructuring. Several authors have suggested possible mechanisms such as surface instability and self-organization, ${ }^{2,15}$ a change in refractive index, ${ }^{4}$ the second harmonic generation, ${ }^{5,12}$ and Coulomb explosion. ${ }^{6}$ However, we have found that these proposed processes are not applicable to explain the previous results for hard thin films reported by Yasumaru et al. ${ }^{3,9}$ and the present authors. ${ }^{16,17}$ In the recent experimental studies, we have shown that the nanostructure formation on the DLC is certainly preceded by the change in bonding structure from DLC to glassy carbon (GC). ${ }^{16,17}$ The GC layer was observed to swell on the surface, and the nanostructuring started on the swelled GC surface. Based on the results of reflectivity measurements for the DLC, we have proposed that nanoscale ablation for the nanostructure formation is induced with a localized field on the film surface. ${ }^{17}$

In this letter, we report an experimental study of the nanostructuring mechanism on the surface, using the DLC film of which surface is patterned with parallel stripes of submicrometer size. The results have demonstrated that the nanoscale ablation was preferentially initiated and developed on the stripe where the localized nanoscale field would be

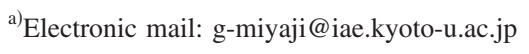

enhanced with the high surface curvature along the direction of the laser polarization.

For the present experiment, we prepared Si substrates on which Si stripes were fabricated with electron-beam lithography and lift-off process. Figures 1(a) and 1(b) show the scanning electron microscope (SEM) images of the patterned Si substrate and an array of stripes in the central part, respectively. The width, length, and height of a single Si stripe are $0.1 \mu \mathrm{m}, 4 \mu \mathrm{m}$, and $50 \mathrm{~nm}$, respectively, and an array consists of 36 parallel stripes drawn at four different periods of 200, 400, 800, and $1600 \mathrm{~nm}$, as seen in Fig. 1(b). The four arrays in the central target area of $50 \times 50 \mu \mathrm{m}^{2}$ are set in a cross shape so that the stripes are oriented to both directions parallel and perpendicular to the linearly polarized $E$-field of incident laser pulses. The eight square-box-shape stripes surrounding the crossed arrays have been drawn for the sake of finding and monitoring the patterned target area with a charge coupled device camera, since those work as a grating for the visible light. Figures 1(c) and 1(d) are the SEM and scanning probe microscope (SPM) images of two stripes coated with DLC, respectively. The DLC of $900 \mathrm{~nm}$ in thickness was deposited on the patterned $\mathrm{Si}$ substrate with a plasma-based ion implantation system, and then the DLCcoated stripe was $\sim 500 \mathrm{~nm}$ in width and $\sim 100 \mathrm{~nm}$ in height, (a)

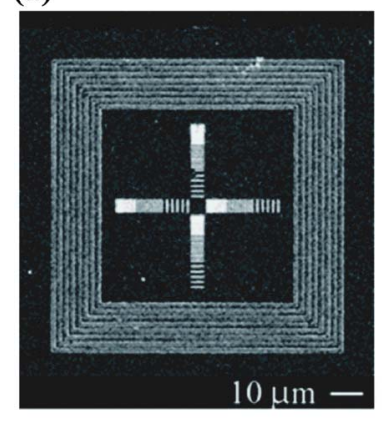

(b)

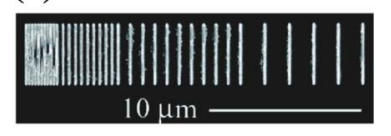

(c)
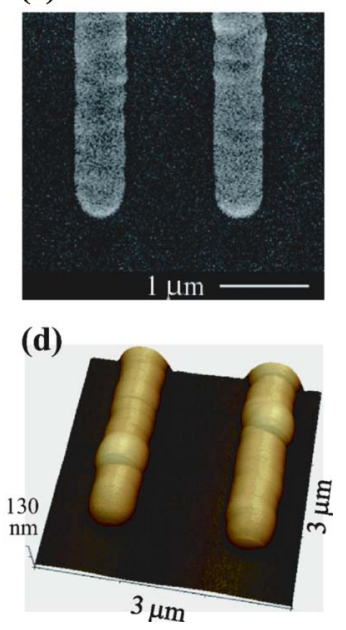

FIG. 1. (Color online) SEM images of (a) the patterned Si substrate, (b) an array of stripes in the central area, (c) the DLC-coated stripes at $800 \mathrm{~nm}$ period, and (d) SPM image of the same stripes as in (c). 


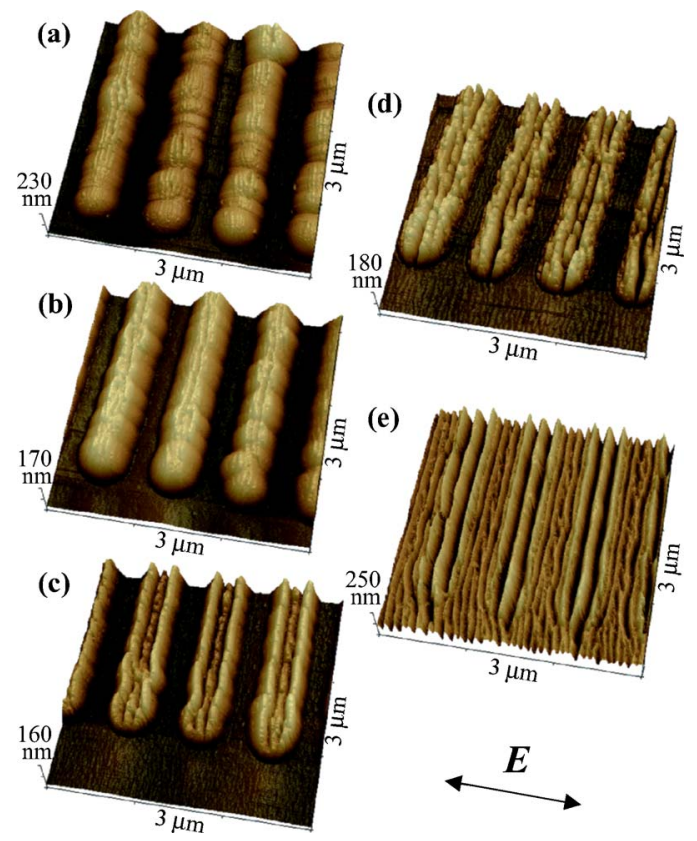

FIG. 2. (Color online) SPM images of the $800 \mathrm{~nm}$ period DLC stripes irradiated with (a) $N=100$, (b) $N=200$, (c) $N=300$, (d) $N=500$, and (e) $N$ $=1000$ at $F=70 \mathrm{~mJ} / \mathrm{cm}^{2}$. The arrow denotes the polarization direction.

as shown in Fig. 1(d). The coated stripes were well isolated at 800 and $1600 \mathrm{~nm}$ periods, whereas those at 200 and $400 \mathrm{~nm}$ periods were partially overlapped with each other to have a reduced height of the stripes.

For the ablation experiment, we used a Ti:sapphire laser system delivering $800 \mathrm{~nm}$ and $100 \mathrm{fs}$ pulses at a repetition rate of $10 \mathrm{~Hz}$. The linearly polarized femtosecond pulse was focused in air at normal incidence on the DLC surface with a lens of $1000 \mathrm{~mm}$ focal length. The intensity distribution of the laser beam could be fitted well to the lowest-order Gaussian shape. The focal spot size $w_{0}$ was $100 \mu \mathrm{m}$ in radius at $1 / e^{2}$ of the maximum intensity, and the focused beam was able to cover the whole patterned target area of 50 $\times 50 \mu \mathrm{m}^{2}$. The nanostructuring was observed to be very sensitive to the spatial intensity distribution or to the local intensity in the focused beam. In the analysis of the experimental results, therefore, we derived the spatial fluence $F(r)$ at position $r$ in the focused beam, using the Gaussian distribution $F=F_{0} \exp \left(-2 r^{2} / w_{0}^{2}\right)$, where $F_{0}$ is the maximum fluence at the beam center. In the present experiment, $F$ was $60-150 \mathrm{~mJ} / \mathrm{cm}^{2}$ to be less than the single-pulse ablation threshold of DLC. The ablation was induced by the superimposed number of laser pulses $N=10-1000$. The surface morphology was observed with the SEM and/or SPM.

Figure 2 shows the SPM images of DLC surface including the $800 \mathrm{~nm}$ period stripes irradiated at different $N$ for $F=70 \mathrm{~mJ} / \mathrm{cm}^{2}$. Comparing with the SPM image of stripe surface shown in Fig. 1(d), Fig. 2(a) represents the narrow linelike ablation traces that start to be formed in a small area along the crest of stripes. The mean spacing $l$ between the linelike traces or the periodicity of nanostructure on the stripe is measured to be $l \sim 60 \mathrm{~nm}$. The nanostructure is certainly formed along the direction perpendicular to the laser polarization or parallel to the stripe. For $N=200$, the small structure grows up on the ridge of stripe, as seen in Fig. 2(b). No structure was observed yet on the nonpatterned flat surface outside the crossed stripe arrays. With a further increase in $N$ to 300 , the mean spacing increases to $l \sim 110 \mathrm{~nm}$, while Downloaded 05 Mar 2008 to 130.54 .110 .22 . Redistribution subject

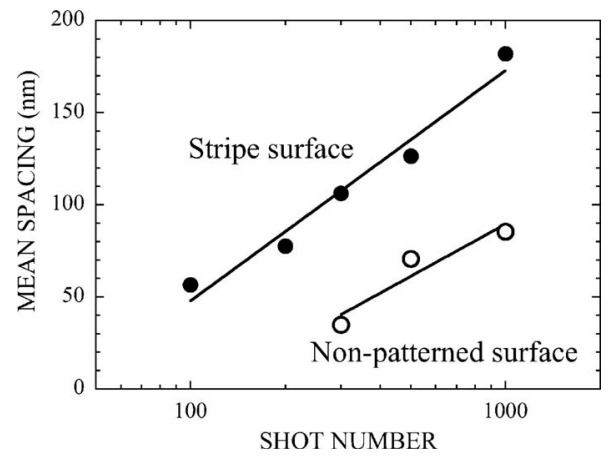

FIG. 3. Mean spacing in the periodic nanostructure formed on the stripe surface (solid circles) and on the nonpatterned surface (open circles) as a function of $N$ at $F=70 \mathrm{~mJ} / \mathrm{cm}^{2}$. The laser $E$-field is perpendicular to the stripe on its surface.

the small ablation traces of $l \sim 40 \mathrm{~nm}$ start to be formed on the nonpatterned surface, as seen in the lower part of Fig. 2(c). With increasing $N$ up to 500-1000, as shown in Figs. 2(d) and 2(e), the ablation traces on the stripe ridge become deeper with the increased spacing of $l \sim 180 \mathrm{~nm}$, while the smaller structure with $l \sim 90 \mathrm{~nm}$ is created on the nonpatterned surface. It is noted that in the area between two stripes the nanostructure grows almost together with that on the nonpatterned surface. These results shown in Fig. 2 strongly suggest that the electric field intensity is locally enhanced to initiate the nanometer-size ablation on the stripe ridge, while the laser $E$-field is kept below the single-pulse ablation threshold. Such enhancement of $E$-field intensity could preferentially be induced due to the high surface curvature along the laser polarization direction. In this case, a small enhancement would be enough for initiating the local ablation, as observed in our pump-probe experiment. ${ }^{17}$

To see quantitatively the effect of the stripe on nanostructuring, we measured the mean spacing $l$ in the nanostructure as a function of $N$. Figure 3 compares the results on the stripe with those on the nonpatterned surface outside the crossed stripe arrays, where the former data are concerned with the stripes at the 800 and $1600 \mathrm{~nm}$ periods. It is clearly seen that the nanostructure is formed efficiently on the stripe, while the spacing $l$ increases with an increase in $N$ on both surfaces.

The nanostructure formation on hard thin films has been observed so far to depend on the laser polarization. ${ }^{3,9,16,17}$ In the present experiment, we were able to study the polarization effect from the different point of view of the correlation between the laser $E$-field and stripe directions. The laser $E$-field incident on a target was parallel or perpendicular to the stripes in the crossed arrays, as mentioned before. Then we could observe the morphology of the stripe surfaces irradiated with the different polarizations under the same condition of $F$ and $N$. Figure 4 shows an example of the pair of SEM images for the stripes on a single target irradiated with the linearly polarized laser pulses. On both stripes parallel and perpendicular to the laser $E$-field, the linelike ablation traces are certainly formed in the direction perpendicular to the $E$-field. On the other hand, as seen in Fig. 4(a), the deeper nanostructure is efficiently produced with $l$ $\sim 110 \mathrm{~nm}$ on the stripes perpendicular to the laser $E$-field, while the shallower nanostructure is formed with the smaller spacing $l \sim 80 \mathrm{~nm}$ on the stripes parallel to the field, as shown in Fig. 4(b). This indicates that the stripe direction parallel to the laser $E$-field is less effective for the nanostrucAIP license or copyright; see http://apl.aip.org/apl/copyright.jsp 


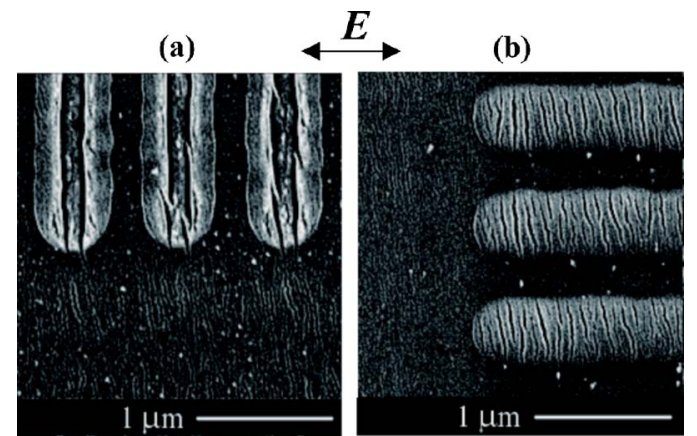

FIG. 4. SEM images of the DLC surfaces including the $800 \mathrm{~nm}$ period stripes (a) perpendicular and (b) parallel to the $E$-field, as indicated by an arrow, with $N=300$ at $F=70 \mathrm{~mJ} / \mathrm{cm}^{2}$.

turing. In addition, as seen in Fig. 4, the shallow structure is created also on the nonpatterned surface outside the stripes, and its structure size is comparable to that on the stripes parallel to the $E$-field. These results are completely consistent with the above picture that the nanoscale ablation is initiated by the efficient enhancement of localized $E$-field, when the incident laser field is perpendicular to the stripe to meet the high surface curvature.

As shown in our previous experiments, ${ }^{17,18}$ the nanostructure formation is preceded by the bonding structure change from DLC to GC. This process swells the surface volume of DLC film, and then the volume expansion may induce some temperature distribution and/or nonuniform stress on the stripe surface to contribute to the nanostructuring process. However, the surface swelling itself is independent of the laser polarization direction, and the resulting ther$\mathrm{mal}$ and/or mechanical effects cannot play a dominant role in the nanostructuring process that strongly depends on the $E$-field direction.

The local field intensity enhanced on the stripe surface is expected to depend on $F$, as well as the surface shape. To see this, we observed the change in surface morphology as a function of $F$ at $N=100$. Figure 5 shows the measured $l$ in the nanostructure formed on the stripe perpendicular to the laser $E$-field and on the nonpatterned surface. The results demonstrate that $F$ required for nanostructuring is greatly reduced on the stripe, compared with $F$ on the nonpatterned surface, as well as $N$ in Fig. 3.

The morphological change of a target surface would certainly be a fingerprint of the nanofield and resulting nanoscale ablation on the surface. ${ }^{19}$ On the basis of the results obtained, we can illustrate the interaction process of nanostructuring on the DLC surface. The volume swelling in the transformation from DLC to GC creates nanometer-size roughness even on a flat DLC surface. ${ }^{17}$ The GC layer with the small bandgap is able to efficiently produce free electrons in the surface through the light absorption, and then the freeelectron density produced would not be uniform in the GC layer on the nanometer level. In the laser field, the free electrons tend to coherently oscillate and localize along the $E$-field direction in the area much smaller than the laser wavelength. The localized electron-hole pairs or the excited surface structure can lead to an enhancement of the evanescent field in the material surface so as to initiate the local ablation, while the laser fluence is less than the single-pulse ablation threshold. The enhancement of local field intensity should be efficient in the direction of high surface curvature

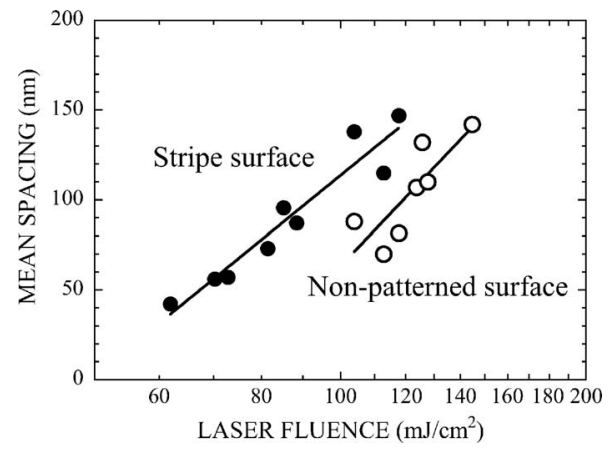

FIG. 5. Mean spacing in the periodic nanostructure formed on the stripe surface (solid circles) and nonpatterned surface (open circles) as a function of $F$ with $N=100$. The laser $E$-field is perpendicular to the stripe on its surface.

along the laser $E$-field. This process would be similar to the enhancement in optical near field. ${ }^{19,20}$

In summary, using the patterned DLC target, we have made the femtosecond-laser ablation experiment at low fluence to study the interaction process responsible for the nanostructuring of DLC film. The results have demonstrated that the nanoscale ablation is preferentially induced on the crest of stripe where the local field intensity would be enhanced with the high surface curvature along the laser $E$-field direction, leading to the conclusive picture that localized nanofield plays a key role in the nanostructuring.

The authors thank S. Oku of NTT-AT, Inc. for his fabrication of the patterned Si substrate, M. Ohtsu for his helpful discussions and suggestions on nanophotonics, A. E. Kaplan for his valuable discussions, and Y. Miyatani for his help in the experiment. This work was partially supported by the Grant-in-Aid for Scientific Research (A) (18206010).

${ }^{1}$ J. Bonse, H. Sturm, D. Schmidt, and W. Kautek, Appl. Phys. A: Mater. Sci. Process. 71, 657 (2000).

${ }^{2}$ F. Costache, M. Henyk, and J. Reif, Appl. Surf. Sci. 186, 352 (2002).

${ }^{3}$ N. Yasumaru, K. Miyazaki, and J. Kiuchi, Appl. Phys. A: Mater. Sci. Process. 76, 983 (2003)

${ }^{4}$ Q. Wu, Y. Ma, R. Fang, Y. Liao, Q. Yu, X. Chen, and K. Wang, Appl. Phys. Lett. 82, 1703 (2003).

${ }^{5}$ A. Borowiec and H. K. Haugen, Appl. Phys. Lett. 82, 4462 (2003).

${ }^{6}$ Y. Dong and P. Molian, Appl. Phys. Lett. 84, 10 (2004).

${ }^{7}$ P. Rudolf and W. Kautek, Thin Solid Films 453/454, 537 (2004).

${ }^{8}$ G. Daminelli, J. Krüger, and W. Kautek, Thin Solid Films 467, 334 (2004).

${ }^{9}$ N. Yasumaru, K. Miyazaki, and J. Kiuchi, Appl. Phys. A: Mater. Sci. Process. 81, 933 (2005).

${ }^{10}$ J. Bonse, M. Munz and H. Sturm, J. Appl. Phys. 97, 013538 (2005).

${ }^{11}$ X. C. Wang, G. C. Lim, F. L. Ng, W. Liu, and S. J. Chua, Appl. Surf. Sci. 252, 1492 (2005).

${ }^{12}$ T. Q. Jia, H. X. Chen, M. Huang, F. L. Zhao, J. R. Qiu, R. X. Li, Z. Z. Xu, X. K. He, J. Zhang, and H. Kuroda, Phys. Rev. B 72, 125429 (2005).

${ }^{13}$ T. Tomita, K. Kinoshita, S. Matsuo, and S. Hashimoto, Jpn. J. Appl. Phys., Part 2 45, L444 (2006).

${ }^{14}$ Z. Guosheng, P. M. Fauchet, and A. E. Siegman, Phys. Rev. B 26, 5366 (1982).

${ }^{15}$ F. Costache, M. Henyk, and J. Reif, Appl. Surf. Sci. 208/209, 486 (2003).

${ }^{16}$ K. Miyazaki, N. Maekawa, W. Kobayashi, N. Yasumaru, and J. Kiuchi, Appl. Phys. A: Mater. Sci. Process. 80, 17 (2005).

${ }^{17}$ G. Miyaji and K. Miyazaki, Appl. Phys. Lett. 89, 191902 (2006).

${ }^{18} \mathrm{~N}$. Yasumaru, K. Miyazaki, J. Kiuchi, and H. Magara, Proc. SPIE 5662, 755 (2004).

${ }^{19}$ A. Plech, V. Kotaidis, M. Lorenc, and J. Boneberg, Nat. Phys. 2, 44 (2006).

${ }^{20}$ M. Ohtsu and K. Kobayashi, Optical Near Fields (Springer, Berlin, 2004). 